\title{
Multidisciplinary Views of Business Contracts
}

\author{
Munindar P. Singh ${ }^{1}$ and Nirmit Desai ${ }^{2}$ \\ 1 North Carolina State University, Raleigh, NC 27695-8206, USA \\ 2 IBM Research, Bangalore 560071, India
}

Several major trends in the services industry drive toward an increasing importance of contracts. These include the formalization of business processes across the client and the provider organizations; resource administration in cloud computing environments; service-level agreements as they arise in infrastructure and networking services; and services viewed from the perspective of real-life engagements.

Previous work on e-commerce architectures and on technical services (such as web and grid services) has focused on low-level aspects of computation. In contrast, the emerging trend toward contracts recognizes the importance of highlevel, flexible specifications of interactions. It is clear that contracts make sense only when the contracting parties are autonomous. Further, contracts help shield the organizational heterogeneity of the contracting parties from one another. In this manner, contracts provide a natural match for a variety of real-world problems that service scientists are beginning to recognize.

By developing and studying contracts from perspectives of multiagent systems, legal and jurisprudence literature, and economics, we would not only find compelling new applications for the concepts and techniques of multiagent systems but also discover new and multidisciplinary methods driven by contracts for architecting service systems.

This tutorial introduces the key ideas and techniques of contracts as they arise in major real-world service engagements; the benefits of formalizing contracts to realize them computationally; and the opportunities and challenges contracts offer for researchers and practitioners. We are seeing increasing interest in contracts from the services research community. Recently, several highprofile research projects have been centered on contracts. While most members of the ICSOC community may be familiar with WS-Agreement, Service-Level, Agreements, and Quality of Service, the attendees may benefit from alternative, multidisciplinary, perspectives into contracts such as the theory and practice of multiagent systems, the legal and jurisprudence literatures, and ideas from social sciences and economics. This tutorial eill enable studies of contracts by explaining why contracts are needed, how they can be represented, and which concepts and techniques support contracts naturally. This tutorial will provide the key background, which attendees can use as a launching pad for their investigations. 